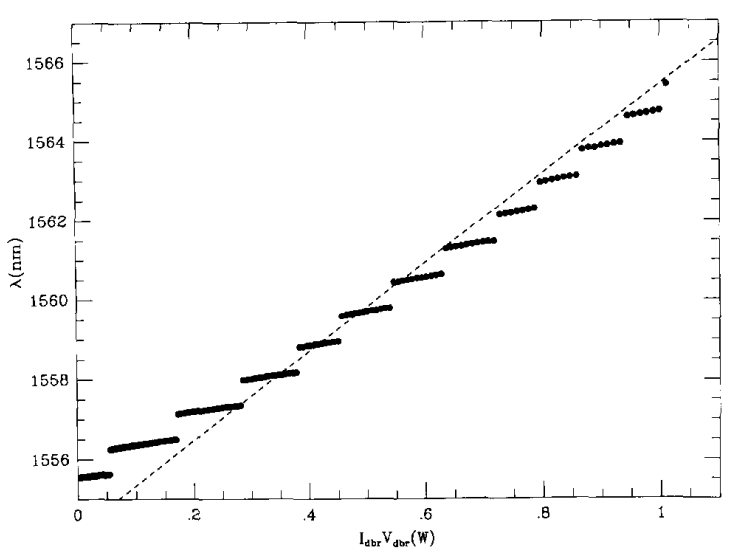

Fig. 3. Wavelength versus tuning power: points show the measured change in $\lambda$ versus $I_{\mathrm{dbr}} V_{\mathrm{dbr}}$ with no voltage applied to the phase-control section. The dashed line is the theoretical curve for the Bragg wavelength, $\lambda_{\mathrm{dbr}}$, versus $I_{\mathrm{dbr}} V_{\mathrm{dbr}}$. The slope of this line was determined using $\zeta=0.02 \mathrm{~m}^{\circ} \mathrm{C} / \mathrm{W}$, which was found by performing a fit to the data in Fig. 2 .

laser is approximately $0.5 \mathrm{~ms}$, which is in good agreement with the results of Öberg et al. [3].

In conclusion, we have demonstrated a DBR laser with a tuning range of over $10 \mathrm{~nm}$. Its behavior is easily modeled. The laser showed only a small degradation in power and linewidth over the entire tuning range. It should find application in WDM systems that require precise wavelength control but do not demand very fast switching speeds.

\section{REFERENCES}

[1] S. Sakano, T. Tsuchiya, M. Suzuki, S. Kitajima, and N. Chinone, "Tunable DFB laser with a striped thin-film heater," IEEE Photon. Technol. Lett., vol. 4, pp. 321-323, Apr. 1992.

[2] J.-M. Verdiell, U. Koren, and T. L. Koch, "Linewidth and alphafactor of detuned-loaded DBR lasers," IEEE Photon. Technol. Lett., vol. 4, pp. 302-325, Apr. 1992.

[3] M. Öberg, S. Nilsson, T. Klinga, and P. Ojala, "A three-electrode distributed Bragg reflector laser with $22 \mathrm{~nm}$ wavelength tuning Tange," IEEE Photon. Technol. Lett, yol. 3 pp. 299-301, Apr. 1991.

[4] T. L. Koch, U. Koren, R. P. Gnall, C. A. Burrus, and B. I. Miller, "Continuously tunable $1.5 \mu \mathrm{m}$ multiple-quantum-well GaInAs/ GaInAsP distributed-Bragg-reflector lasers," Electron. Lett., vol. 24 , no. 23 , pp. $1431-1432$, Nov. 1988.

[5] U. Koren, B. I. Miller, M. G. Young, M. Chien, A. H. Gnauck, P. D. Magill, S. L. Woodward, and C. A. Burrus, "Strained layer multiple quantum well distributed Bragg reflector lasers with a fast-monitoring photodiode," Appl. Phys. Lett., vol. 58, pp. 1239-1240, Mar. 1991.

[6] B. I. Miller, U. Koren, M. G. Young, and M. D. Chien, "Straincompensated strained-layer superlattices for $1.5 \mu \mathrm{m}$ wavelength lasers," Appl. Phys. Lett., vol. 58, pp. 1952-1954, May 1991.

[7] F. Koyama, Y. Suematsu, S. Arai, and T. Tanbun-Ek Tawee, "1.5-1.6 $\mu \mathrm{m}$ GaInAsP/InP dynamic-single mode (DSM) lasers with distributed Bragg reflector," IEEE J. Quantum Electron., vol. QE-19, pp. 1042-1051, June, 1983.

[8] X. Pan, H. Olesen, and B. Tromborg, "A theoretical model of multielectrode DBR lasers," IEEE J. Quantum Electron., vol. QE24, pp. 2423-2432, Dec. 1988.

[9] S. L. Woodward, I. M. I. Habbab, T. L. Koch, and U. Koren, "The side-mode-suppression ratio of a tunable DBR laser," IEEE Photon. Technol. Lett., vol. 2, pp. 854-856, Dec. 1990.

[10] W. B. Joyce and R. W. Dixon, "Thermal resistance of heterostructure lasers," J. Appl. Phys., vol. 46, no. 2, pp. 855-862, Feb. 1975.

\title{
All-Optical Synchronization and Multiplication of the Frequency of Mode-Locked Signals
}

\author{
Paul Phelan, Gerald Farrell, and John Hegarty
}

\begin{abstract}
We report all-optical synchronized multiplication by rational fractions of the modulation frequency of optical signals in self-pulsing two-section semiconductor laser diodes. The effect is based on the overlap of harmonics of the input signal with harmonics of the self-pulsation. We illustrate this new generic function with modelocked inputs rich in harmonics were fractions of 1,2 , and $3 / 2$ are generated, and we identify a number of applications for this effect.
\end{abstract}

QEMICONDUCTOR lasers have been known to self$\checkmark$ pulsate under special conditions especially after some

Manuscript received August 31, 1992.

The authors are with Optronics Ireland Research Centre, Department of Physics, Trinity College, Dublin 2, Ireland.

IEEE Log Number 9204961. catastrophic degradation has occurred. In lasers with multiple CW current inputs through segmented contacts, the self-pulsation can be induced by a proper combination of currents and more importantly, it can be controlled [1]. This self-pulsation has been proposed as a basis for carrying out the function of all-optical clock extraction from data streams [2], [3], and for regenerating data in combination with bistable elements [2]. In two recent papers [4], [5] we have shown that a self-pulsing diode with two current inputs can be synchronized to an input signal whose modulation frequency is a multiple of the self-pulsing frequency. We have shown that this type of frequency division with synchronization is a strong function of the harmonic content of both the modulated signal and the 


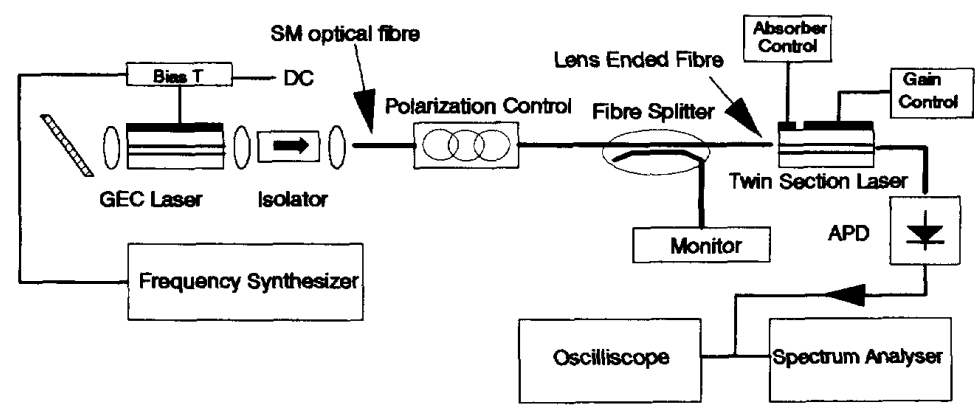

Fig. 1. Experimental setup.

self-pulsing signal. In particular, a mode-locked input gives better synchronization behavior than a sinusoidal input because of greater harmonic overlap. In this letter, we show that this form of synchronization can be generalized to two frequencies which are relational fractions of each other, limited only by the harmonic content. Such a generic effect has the possibility of giving integrated functional devices useful in optical retiming for telecommunications, in clock distribution at several frequencies locked to a master clock, and in time-division multiplexing/demultiplexing. We demonstrate this by showing that fractions of 1,2 , and $3 / 2$ can be generated in one device.

A schematic of the experimental setup is shown in Fig. 1 and is similar to that used in [4] and [5]. The self-pulsing laser has a 4:1 gain-to-absorber length ratio with an overall length of $500 \mu \mathrm{m}$. Self-pulsing occurs for a gain current of $90 \mathrm{~mA}$ and a typical absorber voltage of 0.294 $\mathrm{V}$. The self-pulsing frequency is controllable from about $200-900 \mathrm{MHz}$ by the absorber bias [1]. The modulated source is a tunable (grating external cavity) actively mode-locked laser diode with output pulse widths less than $75 \mathrm{ps}$. The frequency of the pulse train is determined by the external cavity length, to which the electronic frequency synthesizer is tuned. The output from the synthesizer is amplified before being applied to the laser chip through a bias $T$, to actively mode-lock the tunable laser. The optical output from this source is passed through an isolator and coupled into a single-mode fiber. This signal is then passed through a polarization controller, and a fiber-splitter, before being injected into the absorber section of a two-section laser.

To achieve synchronization and multiplication injection locking [6] of the self-pulsing laser to the signal laser is performed, as suggested previously [4], [5]. To do this the signal laser was spectrally tuned to overlap a longitudinal mode of the self-pulsing laser. Injection locking can be observed as a collapse of the multilongitudinal mode structure of the self-pulsing laser to one or two dominant modes. After careful adjustment of the input polarization to match the two polarization modes, a power input of a few microwatts was sufficient to induce locking [5]. After injection locking, synchronization was initially achieved by tuning the signal frequency and self-pulsing frequency to overlap each other giving 1:1 synchronization. This was achieved by adjusting the absorber bias so that the selfpulsing frequency was close to the signal frequency with no input ( $440 \mathrm{MHz}$ in this case).

The most sensitive method to measure the degree of synchronization is the RF spectrum of the self-pulsing laser output as shown in Fig. 2 for no injection (a) and for the case of an injected signal (b). These spectrum were taken using a $1.5 \mathrm{GHz}$ APD and a $20 \mathrm{GHz}$ spectrum analyzer. After synchronization has been achieved the absorber bias can be adjusted accurately to minimize the noise floor level and optimize synchronization. The full width at half maximum of the main peak decreased from $130 \mathrm{KHz}$ to less than $14 \mathrm{~Hz}$ after injection and the peak height relative to noise floor increased to greater than 50 dB. Fig. 3 shows oscilloscope traces of the input signal (a) and the self-pulsing laser output (b), illustrating the synchronization in time. The synchronized average light output power was typically $19 \mathrm{~dB}$ greater than the input signal power demonstrating that significant amplification is also taking place. The input pulse length, measured using a $20 \mathrm{GHz}$ photodiode, was less than 75 ps.

We now show that in this setup we can achieve multiplication of the input frequency by an integer or by a rational fraction. Previously, we showed that by tuning the input fundamental frequency to a harmonic of the selfpulsing laser frequency, integer division was possible as a result of an overlap of the fundamental frequency of the self-pulsing laser and a higher harmonic of the signal laser [4], [5]. If any of the harmonics overlap each other then synchronization should also be possible even if there is no overlap at the fundamental frequencies. To show this, we tuned the self-pulsing frequency to twice the signal frequency and obtained oscilloscope traces shown in Fig. 4. In this figure, the lower trace is the input pulse train while the top trace is the output from the self-pulsing laser at an input power of $9.2 \mu \mathrm{W}$. The oscilloscope is triggered off the frequency synthesizer used to generate the input signal, showing clearly that synchronization is occurring. The self-pulsing frequency in this case was pulled from its free-running value of $875 \mathrm{MHz}$ to $880.06 \mathrm{MHz}$, exactly twice the input signal frequency. We have similarly seen multiplication by 3 . The power input required is higher in this case because of the decrease in the power of the signal laser at its third harmonic. The degree of integer 


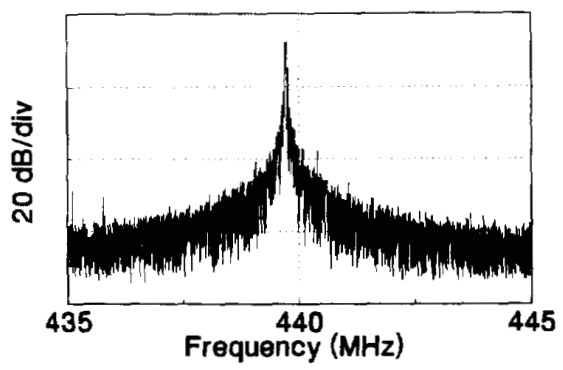

(a)

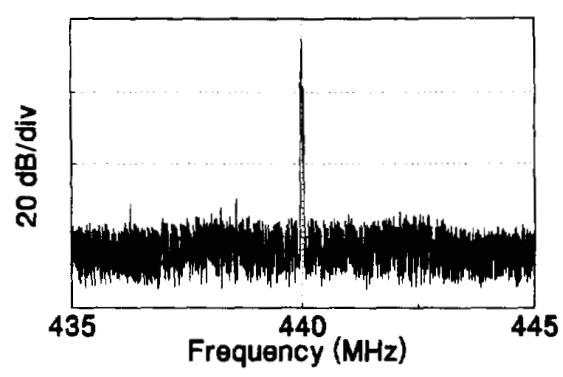

(b)

Fig. 2. Synchronization of modelocked signals, as illustrated by the RF spectrum. Trace (a) is the free-running (unsynchronized) output of the self-pulsing laser while trace (b) is the synchronized output.

(b)

(a)

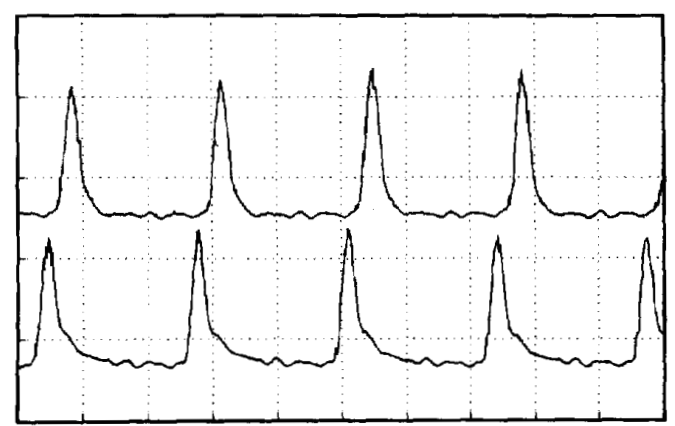

\section{$1 \mathrm{~ns} / \mathrm{div}$}

Fig. 3. Synchronization in time illustrated by oscilloscope traces. Trace (a) is the input mode-locked signal, while trace (b) is the synchronized output of the self-pulsing laser.

multiplication $N$ is limited by the power in the harmonics of the input signal and the power in the self-pulsing harmonics which resonate. For mode-locked pulses we expect $N>10$.

By overlapping a harmonic of the input signal frequency with a harmonic of the self-pulsing frequency, multiplication by a rational fraction can be performed. To demonstrate this function the grating laser was again modelocked at $440.03 \mathrm{MHz}$, while the self-pulsation frequency was adjusted to about $660 \mathrm{MHz}$. In this case the third harmonic of the mode-locked laser overlaps with the second harmonic of the self-pulsing laser. Fig. 5 illustrates (b)

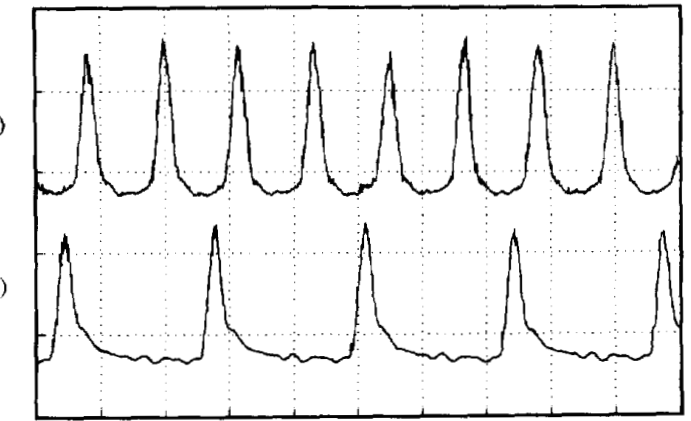

\section{$1 \mathrm{~ns} / \mathrm{div}$}

Fig 4. Oscilloscope traces illustrating multiplication by two. Trace (a) is the input signal from the grating laser, trace (b) is the synchronized output of the self-pulsing laser (at a frequency equal to the input signal frequency multiplied by 2 ).

(b)

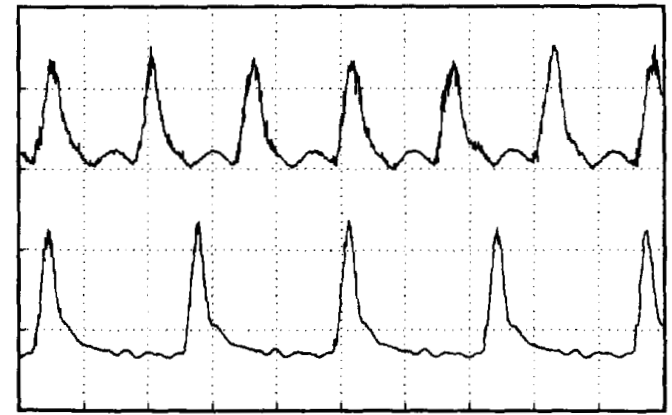

\section{$1 \mathrm{~ns} / \mathrm{div}$}

Fig. 5. Oscilloscope traces illustrating multiplication by $3 / 2$. Trace (a) is the input signal from the grating laser, while trace (b) is the synchronized output of the self-pulsing laser (at a frequency equal to the input frequency multiplied by $3 / 2$ ).

synchronised multiplication by $3 / 2$; the upper trace is the self-pulsing laser output and the lower trace is the input signal frequency. The input power required is similar to that necessary for multiplication by 2 . The additional jitter observed on the output signal in Fig. 5(b) is due to the method used to trigger the oscilloscope. For this case the synthesizer cannot be used as a trigger, since the self-pulsing output frequency is 1.5 times the input signal frequency. Therefore, the electrical signal from the APD is split before the oscilloscope input, and is used for triggering itself, thereby introducing additional jitter. Other rational fractions such as $2 / 3$ have also been observed, and in principle any rational fraction should be obtainable subject to sufficient power in the overlapping harmonics. The limit on the number of fractional frequencies that can be generated is again determined by the harmonic content. Fractions with low denominators will be most favored as they correspond to overlap of lower, stronger harmonics.

The ability to generate new frequencies from a master 
reference frequency is potentially useful in several applications. In clock distribution in electronic circuits, the shorter the pulse the more accurate is the timing. Short pulses also increase the harmonic content leading to a greater range of frequencies that can be generated [7]. It is possible to produce shorter pulses from a self-pulsing two section laser by controlling the carrier lifetimes, or by coupling to an external cavity [8], while modelocking of the master laser can, in principle, give subpicosecond pulses. In time-division multiplexing, frequency multiplication by a large number may sometimes be necessary in which case the self-pulsing laser should have a pulse length short enough to have sufficient harmonic content at the signal frequency for synchronization. Since the self-pulsing frequency can be controlled by the absorber bias, it is possible with the same device to switch from one output frequency to another under voltage control, thereby producing an optical frequency synthesizer.

In summary, we have demonstrated all-optical synchronized multiplication of the frequency of an input modelocked laser signal to a self-pulsing two-section laser diode. Multiplication by integers and rational fractions have been demonstrated. We believe that this is the first demonstration of generic frequency conversion using diode lasers and we expect it to have several applications including clock distribution, high-speed time-division multiplexing/demultiplexing, and optical frequency synthesis.

\section{ACKNOWLEDGMENT}

We would like to thank Dr. M. Robertson of BT Laboratories for providing the laser diodes used in this work.

\section{REFERENCES}

[1] G. Farrell, P. Phelan, and J. Hegarty, "Self-pulsation operating regime for absorber of twin section laser diode," Electron. Lett., vol. 27, pp. 1403-1404, 1991.

[2] M. Jinno and T. Matsumoto, "Optical retiming regenerator using $1.5 \mu \mathrm{m}$ multi-electrode DFB LDs," Electron. Lett., vol. 25, pp. $1332-1333,1988$

[3] P. Barnsley and H. Wickes, "All optical clock recovery from 2.5 $\mathrm{Gb} / \mathrm{s}$ NRZ data using a self pulsating $1.56 \mu \mathrm{m}$ laser diode," Electron. Lett., vol. 28, pp. 4-5, 1992.

[4] G. Farrell, P. Phelan, and J. Hegarty, "All-optical synchronization with frequency division using a self-pulsating laser diode," Electron. Lett., vol. 28, pp. 738-739 1992.

[5] P. Phelan, G. Farrell, and J. Hegarty, "All-optical synchronization and frequency division of modelocked pulses," IEEE Photon. Technol. Lett., vol. 4, pp. 930-932, Aug. 1992.

[6] R. Lang, IEEE J. Quantum Electron., vol. QE-18, pp. 976-983, 1982.

[7] G. Farrell, P. Phelan, and J. Hegarty, "All-optical clock distribution with synchronous frequency division and multiplication," Electron. Lett., vol. 28, pp. 1387-1388, 1992.

[8] K. Lau, J. Lightwave Technol., vol. 7, pp. 400-419, 1989.

\title{
Interferometric Measurement of the Linewidth Enhancement Factor of a 1.55 $\mu \mathrm{m}$ Strained Multiquantum-Well InGaAs/InGaAsP Amplifier
}

\author{
J. Ehrhardt, A. Villeneuve, G. I. Stegeman, H. Nakajima, J. Landreau, and A. Ougazzaden
}

\begin{abstract}
The linewidth enhancement factor of an InGaAs / InGaAsP strained multiquantum well optical amplifier was measured interferometrically. It varied from 3 to 18 over the wavelength range from 1500 to $1600 \mathrm{~nm}$ with injection currents varying from one to four times the lasing threshold of the uncoated device. A rate equation model gave differential gain

Manuscript received July 7, 1992; revised September 8, 1992. This work was supported by AFOSR-91-0086.

J. Ehrhardt is with CREOL, University of Central Florida, Orlando, FL 32826 on leave from ESIEE B.P. 9993162 Noisy-le-Grand, France.

A. Villeneuve, and G. I. Stegeman are with CREOL, University of Central Florida, Orlando, FL 32826.

H. Nakajima, J. Landreau, and A. Ougazzaden are with Centre National d'Etudes des Telecommunications, Laboratoire de Bagneux, France Telecom, 92220 Bagneux, FRANCE.

IEEE Log Number 9204083.
\end{abstract}

and refractive index change per carrier, respectively, in the range 0.3 to $2.5 \times 10^{-15} \mathrm{~cm}^{2}$ and -5 to $-8 \times 10^{-20} \mathrm{~cm}^{3}$.

\section{INTRODUCTION}

$\mathrm{T}$ HE linewidth enhancement factor $(\alpha)$, differential gain, and index change per injected carrier are the key parameters needed to evaluate the performance of semiconductor optical amplifiers in communications systems and photonic switching. Previous theoretical and experimental work has shown that strained quantum well devices have a low $\alpha$, high differential gain and refractive index [1]-[7]. The techniques used to measure these properties apply principally to lasers and lead to spectral 